\title{
Indexing Baselines: Determining physiological stress in res- cued orphaned koala joeys under rehabilitation by comparing faecal cortisol levels between healthy and impaired individuals
}

\author{
Harsh K. Pahuja ${ }^{1}$ and Edward J. Narayan ${ }^{1,3 *}$ \\ 1 School of Agriculture and Food Sciences, Faculty of Science, The University of Queensland, \\ St Lucia, Brisbane, Queensland 4072, Australia. \\ 3 Queensland Alliance for Agriculture and Food Innovation, The University of Queensland, \\ St Lucia, Brisbane, Queensland 4072, Australia \\ * Correspondence: e.narayan@uq.edu.au; Tel.: +61-040-169-7287
}

Publisher's Note: MDPI stays neutral with regard to jurisdictional claims in published maps and institutional affiliations.

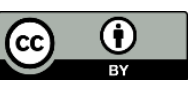

Copyright: () 2021 by the authors. Submitted for possible open access publication under the terms and conditions of the Creative Commons Attribution (CC BY) license (http://creativecommons.org/licenses/by/4.0/).

Simple Summary: Koala (Phascolarctos cinereus) is an iconic marsupial native to Australia, but is vulnerable to high mortality when admitted to wildlife hospitals. As a result, a large number of koala joeys need to be hand reared at rehabilitation centres. The hypothalamic-pituitary-adrenal (HPA) axis plays a vital role in mediating stress (by producing glucocorticoids such as cortisol) associated with inherently stressful environments of rehabilitation centres. However, there are no studies quantifying glucocorticoids in koala joeys under rehabilitation. In this study, we use minimally invasive techniques to quantify faecal cortisol metabolites (FCM) in seven orphaned rescued koala joeys undergoing rehabilitation. Sampling occurred every morning during routine cage cleaning. We use the iterative baseline technique to calculate baseline and peak values of FCM concentrations in each of the seven joeys. Results of this study indicated that there was significant difference in FCM concentrations of joeys and their associated health conditions, with healthy joeys indicating relatively low and consistent FCM concentrations, whereas, diseased joeys indicated a significant increase in FCM concentrations over the sampling period. The lowest baseline and peak FCM concentration recorded was $14.11 \mathrm{ng} / \mathrm{g}$ and $25.65 \mathrm{ng} / \mathrm{g}$ respectively, for a healthy individual, whereas, the highest baseline and FCM concentration was $51.10 \mathrm{ng} / \mathrm{g}$ and $56.58 \mathrm{ng} / \mathrm{g}$ respectively, for a diseased individual. This is the first report quantifying baseline and peak concentrations of cortisol in koala joeys under rehabilitation.

Abstract: Koala (Phascolarctos cinereus) is an iconic folivorous marsupial native to the sclerophyll forests and woodlands of Australia. Due to the ever changing habitat, this species is highly vulnerable to anthropogenic factors such as habitat loss and fragmentation, and this is reflected in increasing number of injured and/or diseased koalas over the years. Majority of adult koalas admitted at wildlife hospitals are deceased, either due to natural causes, or have to be euthanized. Thus, orphaned koala joeys constitute a substantial number of wildlife rescues, and mortality is also prevalent in koala joeys being hand reared/rehabilitated, with little knowledge about the causes of such high rates of mortality. Wildlife hospitals/rehabilitation centres are inherently stressful, and although the hypothalamic-pituitary-adrenal (HPA) axis plays a vital role in mediating the stress endocrine function (by producing glucocorticoids such as cortisol), there are no studies quantifying glucocorticoids in koala joeys. To contribute to this dearth of research, we sampled a total of seven individuals residing at Port Macquarie Koala Hospital, and noted their clinical information. Faecal samples were collected from all seven koala joeys during routine cage cleaning. In total, 123 faecal samples were collected, processed and analysed for cortisol using enzyme-immunoassay (EIA). We used the iterative baseline approach to determine baseline and peak concentrations of FCM in koala joeys. Baseline concentrations ranged between $14.11 \mathrm{ng} / \mathrm{g}$ - $51.10 \mathrm{ng} / \mathrm{g}$ (healthy - sick), whereas, peak FCM concentrations ranged between $25.65 \mathrm{ng} / \mathrm{g}-56.58 \mathrm{ng} / \mathrm{g}$ (healthy - sick). There was a significant difference $(\mathrm{p}<0.05)$ between FCM concentrations of healthy and impaired individuals. Healthy individuals displayed relatively consistent FCM concentrations, whereas, diseased individuals displayed a significant increase in FCM concentrations over time. Our study provides the first 
record of baseline and peak FCM concentrations in rescued koala joeys with their associated clinical condition. Future studies can use the iterative baseline approach to determine FCM concentration in wild koala joeys that can serve as a baseline to compare glucocorticoid levels of rescued joeys.

Keywords: cortisol; faeces; glucocorticoids; iterative baseline; joey; koala; stress

\section{Introduction}

Wildlife around the world are facing ever-increasing coercion from anthropogenic induced environmental factors, resulting in pernicious impacts and menacing the future persistence of native fauna in the wild (Charalambous and Narayan, 2020, Molina-López et al., 2011, Schenk, 2017, Garcês et al., 2019, Gallini et al., 2021). Humans are the chief culprits of extreme environmental change, since anthropogenic activities substantially alter ecosystems, resulting in catastrophic impacts on biodiversity (Goudie, 2018). Ecosystems are modified by humans in a variety of ways, including habitat clearing and fragmentation, grazing and cultivation, urbanisation, introduction of invasive species and contributions to global warming (Hautier et al., 2015). For most organisms, such anthropogenic activities may have severe potential ramifications, particularly for those that are strictly dependent on specialised habitat conditions (Parmesan, 2006). In particular, habitat loss and fragmentation (induced due to population growth, increasing urbanisation and agricultural advancement, climate change, sea-level rise and sedimentation, pollution, expansion of roads, and excessive tourism) is the principal factor leading species towards extinction (Pardini et al., 2017, Machado et al., 2017, Rytwinski and Fahrig, 2012, Short and Burdick, 1996, Valiela et al., 2018, Mantyka-pringle et al., 2012, Coverdale et al., 2013, Gaston et al., 2003). In strictly ecological terms, it can be inferred that there is interspecific competition (Crombie, 1947) between humans and wildlife for resources, particularly land.

The koala (Phascolarctos cinereus; Goldfuss 1817) is an iconic folivorous marsupial native to the sclerophyll forests and woodlands of Australia, and is classified as 'Vulnerable' by the International Union for Conservation of Nature (IUCN) due to the aforementioned anthropogenic induced environmental threats (Woinarski and Burbidge, 2020). In addition, diseases such as chlamydia and oxalate nephrosis are also prevalent in koalas requiring clinical care (Speight et al., 2014, Speight et al., 2016, Fabijan et al., 2019). Wildlife retrospective studies from Australia (QLD, NSW and VIC) highlight that over the years more and more koalas are being rescued and admitted to wildlife hospitals for veterinary attention (Burton and Tribe, 2016, Gonzalez-Astudillo et al., 2017, Griffith et al., 2013, Taylor-Brown et al., 2019) and it has been noted that the clinical outcome for majority of koalas admitted at the wildlife hospitals is either natural death or they need to be euthanized due to their critical condition (Taylor-Brown et al., 2019, Burton and Tribe, 2016, Gonzalez-Astudillo et al., 2017). Increased mortality rates are a result of constant exposure to anthropogenic induced environmental factors, that act as 'stressors' and have a deleterious impact on various biological systems of the animal (Narayan and Williams, 2016). Higher mortality of adult koalas causes higher rescues of young orphans (joeys and juveniles) requiring intensive hand-rearing, for example, a recent study by Taylor-Brown et al. (2019) indicated that 'orphaned' individuals were the second leading cause of admission among wildlife. Marsupials alone contributed $>50 \%$ to this category, and among marsupials, koalas were the second largest group (after possums) that were rescued as orphans. At birth, joeys are extremely small ranging from about $2 \mathrm{~cm}$ long and $1 \mathrm{~g}$ in weight. Birth is followed by pouch occupancy for 5-7 months during which joeys are transported by and given sustenance by their mothers until the first year (Kalman and Levigne, 2002, Gipp, 2004). During this extensive dependant period, maternal milk, eucalyptus and 'pap' constitute the diet of joeys (Morris 
et al., 2016, Barker et al., 2013). 'Pap' feeding is of crucial importance to the growth of the immune system and allow the joeys to gain microbial flora which will increase the digestion of poor-quality foliage when the koala is independent (Barker et al., 2013, Polkinghorne et al., 2013). Orphaned joeys in care are not able to mature their immune systems in the same way as they do not have the required maternal interactions which is only available from a mother koala, thus making them particularly vulnerable to diseases (Morris et al., 2016, Barker et al., 2013). When orphaned, of course, this process needs to be replicated by humans and requires extensive care, close proximity and handling. Gipp (2004) indicates that $90 \%$ of orphaned koalas in care do not survive since it is extremely challenging to hand raise koalas (relative to other marsupial species), however, the underlying cause of mortality is unknown.

By converging literature from various fields of research, we predict that high mortality rates are due to chronic levels of stress associated with epigenetic transfer of stress from the mother to joey (Pfeiffer et al., 2018), physiological stress associated with handling (Narayan et al., 2013) and immunosuppression due to chronic stress (Old and Deane, 2000). To our knowledge there is a dearth of research on koala joeys, specifically in terms of quantifying glucocorticoids (GC) to assess physiological stress, since there are no studies quantifying GC's in koala joeys. There is a substantial delay in GC release and gut transit time in koalas because strictly folivorous and consume eucalyptus leaves that are high in fibre content (Keay et al., 2006). As a result of their prolonged gut transit time (excretory lag-time of over 9 days) faecal based hormone monitoring technique is highly suitable for koalas, since the initial faecal sample can provide us an estimate of stress experienced by koalas several days before arrival to the hospital (Narayan and Vanderneut, 2019).

The primary aim of this study was to use non-invasive hormone monitoring techniques to quantify cortisol (primary GC in mammals) by measuring concentrations of faecal cortisol metabolite (FCM) in orphaned koala joeys under rehabilitation. We then applied the iterative baseline approach to determine the baseline and peak levels of FCM for each joey involved in the study, to compare FCM concentrations associated with their clinical condition.

\section{Materials and Methods}

\subsection{Approval}

Research was performed in accordance with relevant guidelines and regulations. Formal approval was granted by The University of Queensland Ethics (ACEC) Committee (approval number: AE000045).

\subsection{Study site, animals and sample size}

The research study was conducted in collaboration with the Port Macquarie Koala Hospital located in Roto House Historic Site, Lord St, Port Macquarie, New South Wales, 2444 (GPS coordinates: $31^{\circ} 26^{\prime} 35^{\prime \prime}$ S, 152 55'11” E). A total of seven koala joeys [namely Maria River Road Jan (MRRJ), Koala Street Keli (KSK), Pixie (P), Kooloonbung Close Trevor (KCT), Warialda Elizabeth (WE), Slade Collin (SC) and Delung Geoff (DG)] were admitted at the Port Macquarie Koala Hospital and were sampled for this study. The total number of faecal samples from all individuals accounted for $\mathrm{N}=123$, with the following number of samples acquired from each individual - MRRJ $(n=59), K S K(n=40)$, $P(n=13), \operatorname{KCT}(n=7), \operatorname{WE}(n=2), S C(n=1)$, and DG $(n=1)$.

\subsection{Faecal sample collection}


Faecal samples were collected from all seven koala joeys admitted to the Port Macquarie Koala Hospital during the period of study. During routine cage cleaning, 1-5 fresh pellets were collected from each koala joey daily at the same time period in the morning to avoid potential influence of circadian rhythms on FCMs. Fresh pellets were identified by intensity of smell, mucous covering and lack of dehydration. Samples were placed into resealable bags, and labelled with the animal's name, date, identification number, and time of sample collection. Samples were stored at $-20^{\circ} \mathrm{C}$, until they were sent to the laboratory on ice via overnight freight. Upon delivery, the fresh faecal samples were immediately frozen to minimize effects of sample age on FCM levels. All samples were analysed within one month of collection.

\subsection{Faecal sample processing and hormone extraction}

Frozen faecal samples were weighed and then dried in an oven at $65^{\circ} \mathrm{C}$ for a $24 \mathrm{~h}$ period, or until completely dried. Once completely dry, the samples were ground into a fine powder using a mortar and pestle. $10 \%$ ethanol was used to clean the mortar and pestle between individual sample processing to avoid cross-contamination. The ground up powder was sifted through a fine mesh strainer to remove all coarse particles. A 0.2 grams $(\mathrm{g})+/-0.001 \mathrm{~g}$ sample of sifted product was weighed out into a labelled test tube and then stored in a $-20^{\circ} \mathrm{C}$ freezer until use.

For hormone extraction, samples were removed from the $-20^{\circ} \mathrm{C}$ freezer and 2 millilitres (mL) of $90 \%$ ethanol solution was added to the test tube. Tubes were vortexed at medium-high speed on an Eppendorf mini-spin centrifuge for a minimum of $30 \mathrm{~s}$ to thoroughly mix the solution. Tubes were then placed into a water bath $\left(>60^{\circ} \mathrm{C}\right)$ for 30 min to allow hormones to dissolve in the solution. Whilst in the bath, tubes were gently shaken to ensure faeces stayed submerged in ethanol and did not spill over the top of the tube. After $30 \mathrm{~min}$, the supernatant was transferred into an Eppendorf tube, closed and then centrifuged at 10,000 revolutions per minute (RPM) for 5 min to separate any solid faecal residue. If the sample looked turbid or had suspended particles, tubes were centrifuged again for 5 minutes and then pipetted into a new and clean labelled Eppendorf tube. Following this, 25 microliters $(\mu \mathrm{L})$ was aliquoted into a new, clean, labelled Eppendorf tube to which $475 \mu \mathrm{L}$ of assay buffer $\left(39 \mathrm{mM} \mathrm{NaH}_{2} \mathrm{PO}_{4}, 15 \mathrm{mM} \mathrm{NaCl}\right.$ and $0.1 \%$ bovine albumin, $\mathrm{pH} 7.0$ ) was added to obtain a final volume of $500 \mu \mathrm{L}$ (or $0.5 \mathrm{~mL}$ ). Tubes were vortexed at medium-high speed on an Eppendorf mini-spin centrifuge for a minimum of $30 \mathrm{~s}$ to ensure mixing of the assay buffer and ethanol extract.

\subsection{Hormone analysis}

The study used commercially available Detect $X{ }^{\circledR}$ Cortisol Enzyme Immunoassay (EIA) Kit from Arbor Assays (K003-H1/H5, Ann Arbor, MI) to estimate FCM from each faecal extract sample. Each faecal extract sample was assayed in duplicate following the manufacturer's protocol. The kit has a cross-reactivity of $100 \%$ with cortisol, $18.8 \%$ with dexamethasone, $7.8 \%$ with prednisolone (1-Dehydrocortisol), $1.2 \%$ with corticosterone and cortisone, and $<0.1 \%$ with other reactants at the $50 \%$ binding point. For quality control, the study re-ran all samples for which the duplicates had a coefficient of variation $(\mathrm{CV})>20$ in the initial assay, and only retained for analyses samples with a $\mathrm{CV}<20$. Each sample was corrected for the dilution factor and the mean concentration of each set of duplicates was expressed as nanograms of cortisol per gram of faeces.

\subsection{Determining baseline and peak FCM concentrations}

The iterative baseline method, as described by Fanson et al. (2017) was used to determine the baseline and peak levels of FCM concentration of all joeys under rehabilitation at Port Macquarie Wildlife Hospital. The mean and standard deviation (SD) was 
calculated using Microsoft Excel 2021@. The baseline levels for each individual were calculated by using an iterative approach, where all concentrations greater than ' (mean +2 $\mathrm{SD})^{\prime}$ are excluded, and the mean of remaining concentrations are considered to indicate the baseline concentration. All excluded concentrations (i.e. > mean + 2 SD) are considered as peaks.

\subsection{Statistical analysis}

Statistical analysis was performed in Microsoft Excel 2021@ using a Single Factor ANOVA to compare the level of significant difference $(p<0.05)$ in hormone levels between different individuals, and within individuals over the duration of the study. Hormone analysis yielded glucocorticoid results represented as cortisol nanogram per gram (ng/g). Faecal glucocorticoid results over the period of data collection were graphed in a box and whisker plot for each koala with more than one faecal sample. Faecal glucocorticoid results for SC and DG were plotted as individual points, since only one sample could be obtained from these individuals.

\section{Results}

\subsection{Indexing baseline and peak FCM concentrations amongst joeys}

By using the iterative baseline approach, the following baseline and peak levels of FCM for each of the seven koala joeys under rehabilitation were determined (Table 1). Statistical analysis indicated that there was a significant difference in FCM concentrations amongst individuals $(\mathrm{p}<0.05)$.

Table 1. Baseline and peak FCM concentrations in koala joeys under rehabilitation at Port Macquarie Koala Hospital.

\begin{tabular}{|c|c|c|c|c|c|c|c|c|c|}
\hline $\begin{array}{c}\text { Koala } \\
\text { name }\end{array}$ & $\begin{array}{c}\text { Sample } \\
\text { count }\end{array}$ & $\begin{array}{c}\text { Minimum } \\
\text { FCM } \\
\text { (ng/g) }\end{array}$ & $\begin{array}{c}\text { Maximum } \\
\text { FCM } \\
\text { (ng/g) }\end{array}$ & $\begin{array}{c}\text { Average } \\
\text { FCM } \\
\text { (ng/g) }\end{array}$ & Variance & SD & 2*SD & $\begin{array}{c}\text { Minimum } \\
\text { peak FCM } \\
\text { (ng/g) }\end{array}$ & $\begin{array}{c}\text { Baseline } \\
\text { FCM } \\
\text { (ng/g) }\end{array}$ \\
\hline MRRJ & 59 & 7.50 & 37.80 & 14.84 & 29.22 & 5.41 & 10.81 & 25.65 & 14.11 \\
\hline KSK & 40 & 7.95 & 43.42 & 15.76 & 38.29 & 6.19 & 12.38 & 28.14 & 15.06 \\
\hline P & 13 & 12.33 & 20.64 & 15.99 & 5.55 & 2.36 & 4.71 & 20.70 & 15.99 \\
\hline KCT & 7 & 13.22 & 54.38 & 28.13 & 202.39 & 14.23 & 28.45 & 56.58 & 23.75 \\
\hline WE & 2 & 35.37 & 42.91 & 39.14 & 28.37 & 5.33 & 10.65 & 49.79 & 39.14 \\
\hline DG & 1 & 46.11 & 46.11 & 46.11 & N/A & N/A & N/A & N/A & 46.11 \\
\hline SC & 1 & 55.10 & 55.10 & 55.10 & N/A & N/A & N/A & N/A & 55.10 \\
\hline
\end{tabular}


The average faecal cortisol results for all individuals and their respective health conditions are presented below (Figure 1). The average FCM concentration for MRRJ (which was a relatively healthy individual) was $14.84 \mathrm{ng} / \mathrm{g}$. The bottom whisker for MRRJ measured $7.50 \mathrm{ng} / \mathrm{g}$, and the top whisker measured $21.38 \mathrm{ng} / \mathrm{g}$. The average FCM concentration for KSK (who had scabby lesions and a fungal skin infection) was $14.29 \mathrm{ng} / \mathrm{g}$. The bottom and top whisker for KSK measured $7.94 \mathrm{ng} / \mathrm{g}$ and $25.19 \mathrm{ng} / \mathrm{g}$ respectively. The average FCM concentration for $\mathrm{P}$ (clinical diagnosis unknown) was $16.16 \mathrm{ng} / \mathrm{g}$, whereas, the bottom whisker measured $12.33 \mathrm{ng} / \mathrm{g}$, and the top whisker measured $20.63 \mathrm{ng} / \mathrm{g}$. The average FCM concentration for KCT (who had an ecto-parasitic infestation) was $26.19 \mathrm{ng} / \mathrm{g}$, whereas, the bottom whisker and top whisker measured $13.21 \mathrm{ng} / \mathrm{g}$ and $54.37 \mathrm{ng} / \mathrm{g}$ respectively. The average FCM concentration for WE (who tested positive for chlamydia) was $39.14 \mathrm{ng} / \mathrm{g}$, whereas, the bottom whisker measured $35.37 \mathrm{ng} / \mathrm{g}$, and top whisker measured $42.90 \mathrm{ng} / \mathrm{g}$. Box-whisker plots could not be created for DG and SC, since only one sample each could be acquired from these individuals. FCM concentration for DG (clinical diagnosis unknown) was $46.11 \mathrm{ng} / \mathrm{g}$, and that for SC (who had a hind-limb femoral fracture) was $55.10 \mathrm{ng} / \mathrm{g}$. A concise health summary for each individual joey was created based on clinical diagnosis and the information provided by the veterinarians which included basic body condition of the joeys, behaviour, and general health condition (see supplementary material - Table S1).

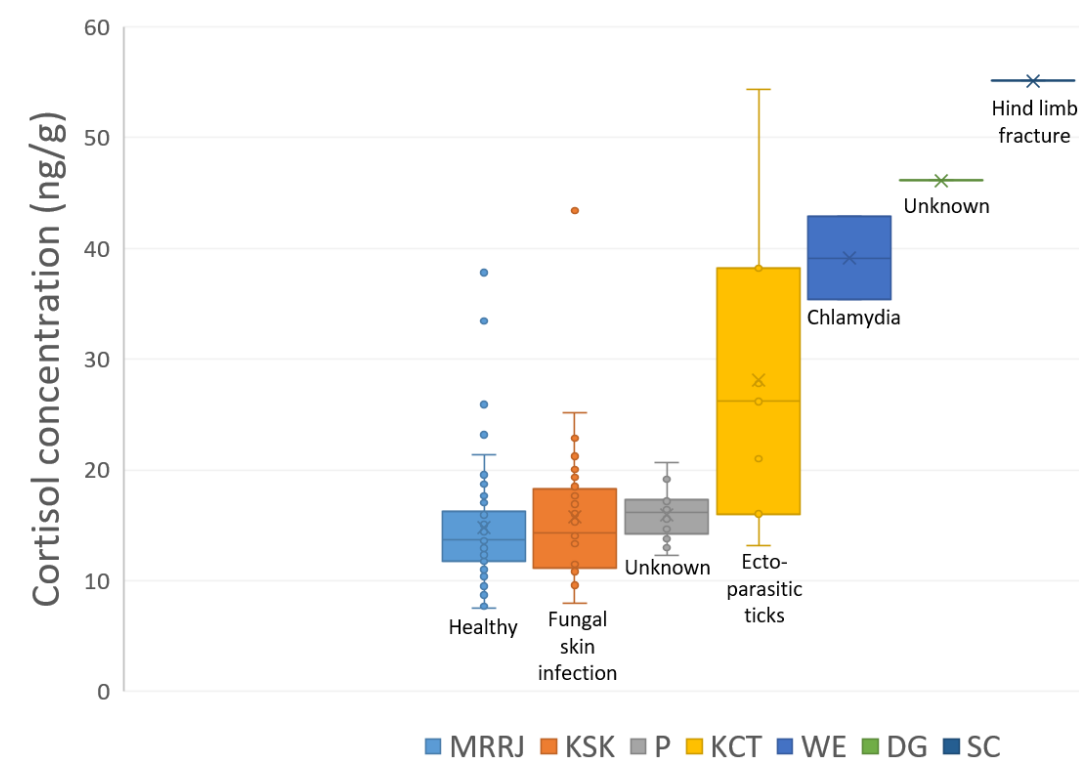

Figure 1. Individual variation and respective health condition for all orphaned koala joeys under rehabilitation at Port Macquarie Koala Hospital.

\subsection{Assessing intra-individual differences in FCM concentrations}

Two individuals, namely MRRJ and KSK, for which extensive longitudinal clinical information was available, were chosen to assess intra-individual differences in FCM concentrations. The rest ( $\mathrm{P}, \mathrm{KCT}, \mathrm{WE}, \mathrm{DG}, \mathrm{SC}$ ) were excluded because there very only a few scat samples obtained from these individuals and there was a dearth of longitudinal clinical information for these individuals. For MRRJ, (who was reported healthy) average FCM concentration were low and relatively consistent, and showed no significant difference $(p>0.05)$ over the study period (Figure 2$)$. 


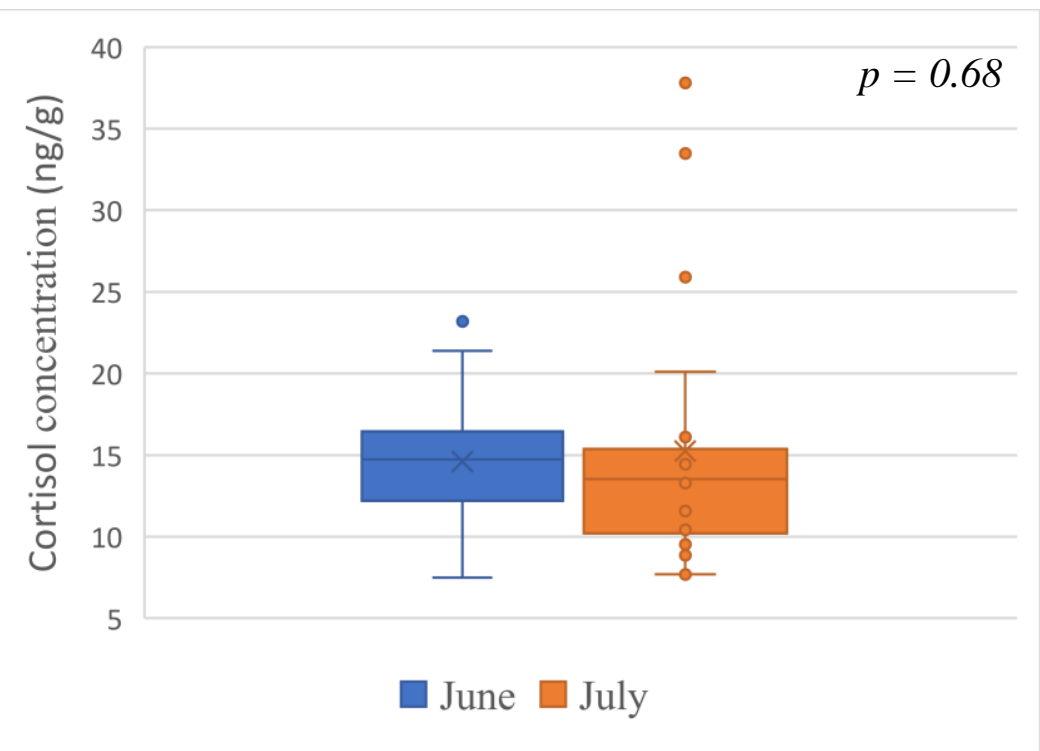

Figure 2. Variation in FCM concentration of MRRJ over sampling period

On the other hand, for KSK (who was reported with a fungal skin infection), average FCM concentration were low and similar to MRRJ initially, but showed a significant increase $(\mathrm{p}<0.001)$ over the study period (Figure 3$)$.

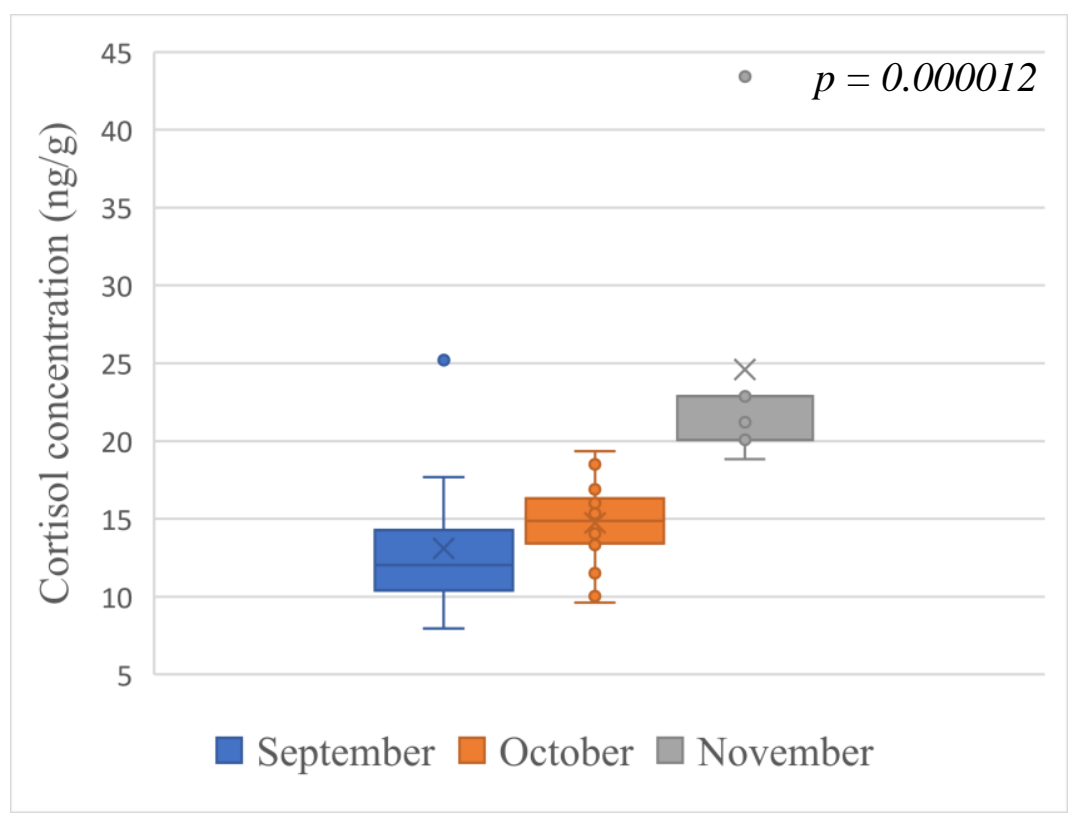

Figure 3. Variation in FCM concentration of KSK over sampling period.

\section{Discussion}

FCM concentration and the characteristic health feature for each of these individuals is illustrated in Figure 1, and it can be noted that there are substantial inter-individual differences based on different health condition of the joeys. MRRJ was brought into the care of Port Macquarie Koala Hospital in March 2019 for eye infection. The faecal samples analysed for FCM quantification for this individual were from June-July 2019, during which the individual was reported to be healthy and with improving body condition. KSK was admitted during September 2019 for fungal skin infection with scabby/crusty lesions on the body. The faecal samples analysed for FCM quantification for this individual were from September-November 2019 and the individual was deceased in April 2020. KCT was admitted during January 2019 with infestation of ecto-parasitic ticks. The faecal samples 
analysed for FCM quantification for this individual were from March 2019. WE was admitted during March 2019 and was tested positive for chlamydia. The faecal samples analysed for FCM quantification for this individual were from the same period. SC was admitted during late December 2019 with femoral fracture and the samples analysed for FCM quantification were from March 2020. Health data for two of seven individuals (P and DG) was unavailable, and thus, excluded from the discussion.

To our knowledge, currently there are no studies that use non-invasive biomarkers such as faeces to quantify GC's as means of assessing physiological stress in koala joeys. As a result, there are no baseline GC concentrations to compare these results with. Using the iterative baseline approach is only a step towards establishing baseline levels of glucocorticoids in rescued koala joeys, however, since rehabilitation sanctuaries are inherently stressful (Charalambous et al., 2021), these indices for rescued joeys are likely to be different relative to their wild-counterparts. It can be noted in Figure 1 that average FCM concentrations for MRRJ are relatively low compared to other individuals. This can be attributed to relatively healthy condition of MRRJ from the period when samples were analysed for FCM quantification. Furthermore, the FCM concentrations for MRRJ were consistent throughout the period of sampling reinforcing the healthy condition of MRRJ (Figure 2). Since MRRJ was admitted in March 2019 and had been a resident of Port Macquarie Koala Hospital since 3 months (until sampling), the effect of stress associated with handling during rehabilitation, can also be ruled out because of potential habituation of the joey to handling, by the staff. Therefore, since MRRJ was the only healthy individual with lowest baseline and peak values (Table 1), FCM concentrations of MRRJ was considered as a baseline for comparison of with other individuals.

Faecal samples for KSK were from the period when it was admitted at the Port Macquarie Koala Hospital and had a fungal skin infection. The average FCM concentration for this individual were similar to MRRJ, however, these values increased substantially over the period of sampling (Figure 3). Furthermore, (a) baseline FCM concentration, (b) maximum FCM concentration recorded, and (c) minimum peak FCM concentration, determined by iterative baseline approach, for KSK, were all higher compared with MRRJ. KSK was deceased in April 2019 after spending 6 months at the hospital. We believe that a significant increase in FCM concentration over time was an indication of sub-clinical symptoms of stress experienced by the joey, and that FCM concentrations would have increased to chronic levels eventually resulting to the death of the joey. Unfortunately, scat samples were not collected from KSK closer to the time of death, and thus, this speculation cannot be confirmed.

However, other individuals (KCT and WE) were also presented with diseases/infections, and depict relatively high concentrations of average FCM (Figure 1) as well as high baseline and peak FCM concentrations, indicating signs of chronic stress. Diseases such as chlamydia, and clinical and sub-clinical cryptococcosis (fungal skin infection), are highly prevalent in koalas (and their joeys), and stress is likely to cause recrudescence of disease even after its been treated (Schmertmann et al., 2018). As explained by Old and Deane (2000), koala joeys do not have an adaptive immune system when they are born and instead, the maturation of immune tissue occurs during the pouch stages, completing the process that is known as 'immune competency'. Since the period of immune competency is abruptly interrupted in orphan koala joeys, they are likely to have a weak immune system, and are thus more vulnerable to diseases. The association of stress with immunosuppression is well established, and further increases the risk of exposure of joeys to diseases (Johnston et al., 2013, McCallum et al., 2018). An adult free-ranging female koala was diagnosed with disseminated cryptococcosis (eventually euthanized), and the joey was hand reared (Schmertmann et al., 2018). At a later stage, routine blood sampling performed on the joey diagnosed it with cryptococcosis but since it displayed no clinical signs, subclinical cryptococcosis was suspected (eventually confirmed) (Schmertmann et 
al., 2018). This serves as just one example for prevalence of vast number of subclinical stressors in koalas (Speight et al., 2016, Patterson et al., 2015, Schmertmann et al., 2018). Since diseases are a prevalent cause of mortality in adult koalas (Charalambous and Narayan, 2020, Gonzalez-Astudillo et al., 2017, Griffith et al., 2013, Burton and Tribe, 2016), and given the potential impact of maternal stress on offspring (Pfeiffer et al., 2018, Stead et al., 2021), it is extremely important to monitor physiological stress in koala joeys under rehabilitation. Future studies assessing physiological stress can verify if sub-clinical symptoms of stress are evident in glucocorticoid concentrations of koala joeys, which can potentially serve as an early detection to prevent mortality in joeys.

\section{Conclusions}

The primary aim of this study was to quantify FCM concentrations in orphaned rescued koala joeys undergoing Koala rehabilitation. It was hypothesised that FCM concentrations between joeys would differ based on their clinical condition. Statistically, there was a significant difference between all joeys, and their faecal cortisol responses associated with their clinical condition. Overall, healthy joey (MRRJ) had low average, low baseline and low peak levels of FCM concentration. Impaired joeys i.e. joeys with diseases (KSK, KCT, WE) had varying levels of FCM concentrations but were relatively higher compared to healthy individuals. Intra-individual variation indicated that healthy individual displayed consistency in FCM concentrations, whereas, diseased individuals displayed a significant increase in FCM concentrations over time, potentially portraying subclinical symptoms of stress.

This study was restricted by the number of faecal samples obtained from certain joeys and the lack of information about clinical diagnosis of certain other joeys. This emphasises the need for rigorous, accurate and detailed data collection. Furthermore, since there are no studies determining glucocorticoid levels in koala joeys, there is no data to compare the baseline results with and the iterative approach to calculate baseline is a step towards indexing baseline glucocorticoid levels in koala joeys. Future studies should focus on indexing glucocorticoid levels in healthy wild koala joeys that can serve as a baseline to compare glucocorticoid levels of rescued joeys.

Supplementary Materials: The following are available online at www.mdpi.com/xxx/s1, Supplementary file 1 .

Author Contributions: Conceptualization, E.N. and H.P.; methodology, E.N. and H.P.; formal analysis, E.N. and H.P.; investigation, E.N. and H.P; resources, E.N.; data curation, E.N. and H.P.; writing - original draft preparation, H.P.; writing - review and editing, E.N. and H.P; supervision, E.N.; project administration, E.N. All authors have read and agreed to the published version of the manuscript.

Funding: This research received external funding from the Australian Koala Foundation (AKF).

Institutional Review Board Statement: The study was conducted according to the guidelines of the Declaration of Helsinki and approved by the Institutional Review Board (or Ethics Committee) of The University of Queensland (approval number: AE000045).

Informed Consent Statement: Not applicable.

Data Availability Statement: Data is contained within the article or supplementary material.

Acknowledgments: We thank the Port Macquarie Koala Hospital for collaboration. We also thank two anonymous reviewers for their valuable comments.

Conflicts of Interest: The authors declare no conflict of interest.

\section{References}


BARKER, C. J., GILLETT, A., POLKINGHORNE, A. \& TIMMS, P. 2013. Investigation of the koala (Phascolarctos cinereus) hindgut microbiome via $16 \mathrm{~S}$ pyrosequencing. Veterinary microbiology, 167, 554-564.

BURTON, E. \& TRIBE, A. 2016. The rescue and rehabilitation of koalas (Phascolarctos cinereus) in Southeast Queensland. Animals, 6, 56.

CHARALAMBOUS, R. \& NARAYAN, E. 2020. A 29-year retrospective analysis of koala rescues in New South Wales, Australia. Plos one, 15, e0239182.

CHARALAMBOUS, R., SIMONATO, T., PEEL, M. \& NARAYAN, E. J. 2021. Physiological stress in rescued wild koalas (Phascolarctos cinereus) being held in a rehabilitation sanctuary: a pilot study. Animals, 11, 2864.

COVERDAlE, T. C., HERRMANN, N. C., ALTIERI, A. H. \& BERTNESS, M. D. 2013. Latent impacts: the role of historical human activity in coastal habitat loss. Frontiers in Ecology and the Environment, 11, 69-74.

CROMBIE, A. C. 1947. Interspecific competition. The Journal of Animal Ecology, 44-73.

FABIJAN, J., CARAGUEL, C., JELOCNIK, M., POLKINGHORNE, A., BOARDMAN, W. S., NISHIMOTO, E., JOHNSSON, G., MOLSHER, R., WOOLFORD, L. \& TIMMS, P. 2019. Chlamydia pecorum prevalence in South Australian koala (Phascolarctos cinereus) populations: Identification and modelling of a population free from infection. Scientific reports, 9, 1-11.

FANSON, K. V., BEST, E. C., BUNCE, A., FANSON, B. G., HOGAN, L. A., KEELEY, T., NARAYAN, E. J., PALME, R., PARROTT, M. L. \& SHARP, T. M. 2017. One size does not fit all: monitoring faecal glucocorticoid metabolites in marsupials. General and comparative endocrinology, 244, 146-156.

GALLINI, S. H., DI GIROLAMO, N., HANN, E., PALUCH, H. \& DIGERONIMO, P. M. 2021. Outcomes of 4819 cases of marine animals presented to a wildlife rehabilitation center in New Jersey, USA (1976-2016). Scientific Reports, 11, 1-7.

GARCÊS, A., PIRES, I., PACHECO, F., FERNANDES, L. S., SOEIRO, V., LÓIO, S., PRADA, J., CORTES, R. \& QUEIROGA, F. 2019. Natural and anthropogenic causes of mortality in wild birds in a wildlife rehabilitation centre in northern Portugal: a ten-year study. Bird Study, 66, 484-493.

GASTON, K. J., BLACKBURN, T. M. \& GOLDEWIJK, K. K. 2003. Habitat conversion and global avian biodiversity loss. Proceedings of the Royal Society of London. Series B: Biological Sciences, $270,1293-1300$.

GIPP, G. 2004. Handraising orphaned koalas. Wildcare Australia [Online]. Available: https://www.awrc.org.au/uploads/5/8/6/6/5866843/nwcc-gipp040726.pdf?fbclid=IwAR03XObcwQZX0VoRVYplB3gRzGfDftG1dWYfetkd7sD5FZQNA2I bC7SYW3s [Accessed 13 August 2021].

GONZALEZ-ASTUDILLO, V., ALLAVENA, R., MCKINNON, A., LARKIN, R. \& HENNING, J. 2017. Decline causes of Koalas in South East Queensland, Australia: a 17-year retrospective study of mortality and morbidity. Scientific reports, 7, 1-11.

GOUDIE, A. S. 2018. Human Impact on the Natural Environment, Newark, UNITED KINGDOM, John Wiley \& Sons, Incorporated. 
GRIFFITH, J. E., DHAND, N. K., KROCKENBERGER, M. B. \& HIGGINS, D. P. 2013. A retrospective study of admission trends of koalas to a rehabilitation facility over 30 years. Journal of Wildlife Diseases, 49, 18-28.

HAUTIER, Y., TILMAN, D., ISBELL, F., SEABLOOM, E. W., BORER, E. T. \& REICH, P. B. 2015. Anthropogenic environmental changes affect ecosystem stability via biodiversity. Science, 348, 336-340.

JOHNSTON, S., BOOTH, R., PYNE, M., KEELEY, T., MACKIE, J., HULSE, L. \& ELLIS, W. 2013. Preliminary study of faecal cortisol and corticosterone as an index of acute cortisol secretion in the koala (Phascolarctos cinereus). Australian veterinary journal, 91, 534-537.

KALMAN, B. \& LEVIGNE, H. 2002. The life cycle of a koala, Crabtree Publishing Company.

KEAY, J. M., SINGH, J., GAUNT, M. C. \& KAUR, T. 2006. Fecal glucocorticoids and their metabolites as indicators of stress in various mammalian species: a literature review. Journal of Zoo and Wildlife Medicine, 37, 234-244.

MACHADO, P. M., SUCIU, M. C., COSTA, L. L., TAVARES, D. C. \& ZALMON, I. R. 2017. Tourism impacts on benthic communities of sandy beaches. Marine Ecology, 38, e12440.

MANTYKA-PRINGLE, C. S., MARTIN, T. G. \& RHODES, J. R. 2012. Interactions between climate and habitat loss effects on biodiversity: a systematic review and meta-analysis. Global Change Biology, 18, 1239-1252.

MCCALLUM, H., KERLIN, D. H., ELLIS, W. \& CARRICK, F. 2018. Assessing the significance of endemic disease in conservation-koalas, chlamydia, and koala retrovirus as a case study. Conservation Letters, 11, e12425.

MOLINA-LÓPEZ, R. A., CASAL, J. \& DARWICH, L. 2011. Causes of morbidity in wild raptor populations admitted at a wildlife rehabilitation centre in Spain from 1995-2007: a long term retrospective study. PLoS One, 6, e24603.

MORRIS, K. M., O’MEALLY, D., ZAW, T., SONG, X., GILLETT, A., MOLLOY, M. P., POLKINGHORNE, A. \& BELOV, K. 2016. Characterisation of the immune compounds in koala milk using a combined transcriptomic and proteomic approach. Scientific reports, 6 , $1-14$.

NARAYAN, E. \& VANDERNEUT, T. 2019. Physiological stress in rescued wild koalas are influenced by habitat demographics, environmental stressors, and clinical intervention. Frontiers in endocrinology, 10, 18.

NARAYAN, E. J., WEBSTER, K., NICOLSON, V., MUCCI, A. \& HERO, J.-M. 2013. Non-invasive evaluation of physiological stress in an iconic Australian marsupial: the Koala (Phascolarctos cinereus). General and Comparative Endocrinology, 187, 39-47.

NARAYAN, E. J. \& WILLIAMS, M. 2016. Understanding the dynamics of physiological impacts of environmental stressors on Australian marsupials, focus on the koala (Phascolarctos cinereus). BMC Zoology, 1, 1-13.

OLD, J. M. \& DEANE, E. M. 2000. Development of the immune system and immunological protection in marsupial pouch young. Developmental \& Comparative Immunology, 24, 445454.

PARDINI, R., NICHOLS, E. \& PÜTTKER, T. 2017. Biodiversity response to habitat loss and fragmentation. Encyclopedia of the Anthropocene, 3, 229-239. 
PARMESAN, C. 2006. Ecological and evolutionary responses to recent climate change. Annu. Rev. Ecol. Evol. Syst., 37, 637-669.

PATTERSON, J. L., LYNCH, M., ANDERSON, G. A., NOORMOHAMMADI, A. H., LEGIONE, A., GILKERSON, J. R. \& DEVLIN, J. M. 2015. The prevalence and clinical significance of Chlamydia infection in island and mainland populations of Victorian koalas (Phascolarctos cinereus). Journal of wildlife diseases, 51, 309-317.

PFEIFFER, J. R., MUTESA, L. \& UDDIN, M. 2018. Traumatic stress epigenetics. Current behavioral neuroscience reports, 5, 81-93.

POLKINGHORNE, A., HANGER, J. \& TIMMS, P. 2013. Recent advances in understanding the biology, epidemiology and control of chlamydial infections in koalas. Veterinary microbiology, 165, 214-223.

RYTWINSKI, T. \& FAHRIG, L. 2012. Do species life history traits explain population responses to roads? A meta-analysis. Biological Conservation, 147, 87-98.

SCHENK, A. 2017. Causes of morbidity and mortality of wildlife species presented to a wildlife clinic in east Tennessee, USA, 2000-2011. Journal of Veterinary Science \& Animal Husbandry, 5.

SCHMERTMANN, L. J., STALDER, K., HUDSON, D., MARTIN, P., MAKARA, M., MEYER, W., MALIK, R. \& KROCKENBERGER, M. B. 2018. Cryptococcosis in the koala (Phascolarctos cinereus): pathogenesis and treatment in the context of two atypical cases. Medical mycology, 56, 926-936.

SHORT, F. T. \& BURDICK, D. M. 1996. Quantifying eelgrass habitat loss in relation to housing development and nitrogen loading in Waquoit Bay, Massachusetts. Estuaries, 19, 730-739.

SPEIGHT, K. N., HAYNES, J. I., BOARDMAN, W., BREED, W. G., TAGGART, D. A., RICH, B. \& WOOLFORD, L. 2014. Plasma biochemistry and urinalysis variables of koalas (P hascolarctos cinereus) with and without oxalate nephrosis. Veterinary clinical pathology, 43, 244-254.

SPEIGHT, K. N., POLKINGHORNE, A., PENN, R., BOARDMAN, W., TIMMS, P., FRASER, T., JOHNSON, K., FAULL, R., BATE, S. \& WOOLFORD, L. 2016. Prevalence and pathologic features of Chlamydia pecorum infections in South Australian koalas (Phascolarctos cinereus). Journal of wildlife diseases, 52, 301-306.

STEAD, S. M., BĂDESCU, I. \& BOONSTRA, R. 2021. Of mammals and milk: how maternal stress affects nursing offspring. Mammal Review.

TAYLOR-BROWN, A., BOOTH, R., GILLETT, A., MEALY, E., OGBOURNE, S. M., POLKINGHORNE, A. \& CONROY, G. C. 2019. The impact of human activities on Australian wildlife. PloS one, 14, e0206958.

VALIELA, I., LLORET, J., BOWYER, T., MINER, S., REMSEN, D., ELMSTROM, E., COGSWELL, C. \& THIELER, E. R. 2018. Transient coastal landscapes: rising sea level threatens salt marshes. Science of the Total Environment, 640, 1148-1156.

WOINARSKI, J. \& BURBIDGE, A. A. 2020. Phascolarctos cinereus. The IUCN Red List of Threatened Species [Online]. Available: https://dx.doi.org/10.2305/IUCN.UK.20201.RLTS.T16892A166496779.en [Accessed 12 August 2021]. 